Pacific Journal of Mathematics

UNITARY EQUIVALENCE OF INVARIANT SUBSPACES OF 


\section{UNITARY EQUIVALENCE OF INVARIANT SUBSPACES OF BERGMAN AND DIRICHLET SPACES}

\section{STEFAN RICHTER}

In this paper we investigate unitary equivalence of invariant subspaces of the Bergman and the Dirichlet space. By definition, this means unitary equivalence of the multiplication operator $M_{z}$ restricted to the invariant subspaces.

We show that no two invariant subspaces of the Bergman space are unitarily equivalent to one another unless they are equal. Under some extra assumption on the invariant subspaces we obtain a similar result for the Dirichlet space.

1. Introduction. Let $T$ be a bounded linear operator on a Hilbert space $\mathscr{H}$. A closed subspace $\mathscr{M}$ of $\mathscr{H}$ is called invariant for $T$, if $T \mathscr{M} \subseteq \mathscr{M}$. The collection of all invariant subspaces of $T$ is denoted by $\operatorname{Lat}(T, \mathscr{H})$.

We shall say that two invariant subspaces $\mathscr{M}, \mathscr{N} \in \operatorname{Lat}(T, \mathscr{H})$ are unitarily equivalent if the operators $T \mid \mathscr{M}$ and $T \mid \mathscr{N}$ are unitarily equivalent, i.e. if there is a unitary operator $U: \mathscr{M} \rightarrow \mathscr{N}$ such that $U T \mid \mathscr{M}=$ $T \mid \mathcal{N} U$.

Some of the operators we are interested in arise as operators of multiplication by $z$ on the spaces $D_{\alpha}, \alpha \in \mathbf{R}$. These operators will in the following be denoted by $\left(M_{z}, D_{\alpha}\right)$. Recall that an analytic function $f$ in the unit disc with Taylor series expansion $f(z)=\sum_{n=0}^{\infty} \hat{f}(n) z^{n}$ is in $D_{\alpha}$ if and only if

$$
\sum_{n=0}^{\infty}(n+1)^{\alpha}|\hat{f}(n)|^{2}<\infty .
$$

The norm on $D_{\alpha}$ is given by $\|f\|_{\alpha}^{2}=\sum_{n=0}^{\infty}(n+1)^{\alpha}|\hat{f}(n)|^{2}$. For $\alpha=$ $-1,0$, or 1 one has the Bergman space $L_{a}^{2}$, the Hardy space $H^{2}$, or the Dirichlet space $D$, respectively.

It follows from Beurling's theorem that all invariant subspaces of the unilateral shift $(\alpha=0)$ are unitarily equivalent to one another. Unitary equivalence of invariant subspaces of $H^{2}$ of the polydisc was investigated by Agrawal, Clark, and Douglas, see [1].

We shall consider two cases, where the situation drastically differs from the $H^{2}$ case. The first one deals with subnormal multiplication 
operators and applies for example to the spaces $D_{\alpha}, \alpha<0$. Theorem 1 will imply that no two different invariant subspaces of the operator $\left(M_{z}, D_{\alpha}\right), \alpha<0$, are unitarily equivalent to one another.

Afterwards we shall discuss unitary equivalence of invariant subspaces of the Dirichlet space $D=D_{1}$. In Theorem 2 we shall show that, if $\mathscr{M}, \mathscr{N} \in \operatorname{Lat}\left(M_{z}, D\right)$ and if either $\mathscr{M}$ contains an outer function or $\mathscr{N} \subseteq \mathscr{M}$, then $\mathscr{M}$ and $\mathscr{N}$ are unitarily equivalent if and only if $\mathscr{M}=\mathscr{N}$.

In $\S \S 2$ and $3 \mathbf{N}$ will denote the set of positive integers, $S^{-}$will be the closure (in the appropriate topology) of the set $S$, and for $f \in \mathscr{H}$, [ $f$ ] denotes the smallest invariant subspace of $M_{z}$ containing $f$.

2. Subnormal multiplication operators. Suppose $\mu$ is a positive, finite, compactly supported Borel measure in the complex plane. By $L^{2}(\mu)$ we denote the space of equivalence classes of $\mu$-square-integrable Borel measurable functions on $\mathbf{C}$. As is usually done, we shall not bother to distinguish between a function $f$ and the equivalence class it represents. Furthermore, we shall assume that the functions in $L^{2}(\mu)$ have the support of $\mu$ as their domain. $N_{z}$ is the normal operator on $L^{2}(\mu)$ defined by $\left(N_{z} f\right)(\lambda)=\lambda f(\lambda)$ for all $f \in L^{2}(\mu) . P^{2}(\mu)$ denotes the closure of the polynomials in $L^{2}(\mu)$.

For the proof of Theorem 1 we shall need a lemma which is certainly well known. However, we were unable to find this exact statement anywhere in the literature.

LEMMA 1. If $\mu$ is as above, then

$$
L^{2}(\mu)=\operatorname{span}\left\{\bar{z}^{n} h: n \in \mathbf{N} \cup\{0\}, h \in P^{2}(\mu)\right\} ;
$$

thus $N_{z}$ is a minimal normal extension of $N_{z} \mid P^{2}(\mu)$.

Proof. Let $\mathscr{L}$ be the set of finite linear combinations of the form $\bar{z}^{n} q$, where $n \in \mathbf{N} \cup\{0\}$ and $q$ is a polynomial, and let

$$
\mathscr{K}=\operatorname{span}\left\{\bar{z}^{n} h: n \in \mathbf{N} \cup\{0\}, h \in P^{2}(\mu)\right\} .
$$

Clearly $\mathscr{L} \subseteq \mathscr{K} \subseteq L^{2}(\mu)$. We shall show that $\mathscr{L}$ is dense in $L^{2}(\mu)$.

It follows from the Stone-Weierstrass theorem that the uniform closure of $\mathscr{L}$ equals $C(K)$, the algebra of all continuous functions on $K$, the support of $\mu$. Using Lusin's theorem we see that the $L^{2}(\mu)$ closure of $C(K)$ equals $L^{2}(\mu)$. Hence $\mathscr{L}^{-}=\mathscr{K}=L^{2}(\mu)$.

Theorem 1. Suppose $\mu$ is a positive, finite, compactly supported Borel measure in the complex plane. Furthermore, assume that the 
support of $\mu$ equals $\Omega^{-}$for some open and connected subset $\Omega$ of $\mathbf{C}$. Let $\mathscr{H}$ be an invariant subspace of $N_{z}$, which consists only of functions analytic on $\Omega$ and set $M_{z}=N_{z} \mid \mathscr{H}$. Then two invariant subspaces $\mathscr{M}, \mathscr{N} \in \operatorname{Lat}\left(M_{z}, \mathscr{H}\right)$ are unitarily equivalent if and only if $\mathscr{M}=\mathscr{N}$.

Proof. Suppose $\mathscr{M}, \mathscr{N} \in \operatorname{Lat}\left(M_{z}, \mathscr{H}\right)$ and $U: \mathscr{M} \rightarrow \mathscr{N}$ is a unitary operator such that $\left(M_{z} \mid \mathscr{N}\right) U=U\left(M_{z} \mid \mathscr{M}\right)$. Suppose $f \in \mathscr{M}$. We have to show that $f \in \mathscr{N}$. This will imply that $\mathscr{M} \subseteq \mathscr{N}$. By symmetry we shall then have proved that $\mathscr{M}=\mathscr{N}$.

We may assume that $f \neq 0$. Set $g=U f$. The restriction of $U$ to $[f]$ is a unitary operator from $[f]$ onto $[g]$ and we have

$$
\int|p|^{2}|f|^{2} d \mu=\int|p|^{2}|g|^{2} d \mu
$$

for all polynomials $p$. Thus we can define a unitary operator

$$
U^{\prime}: P^{2}\left(|f|^{2} d \mu\right) \rightarrow P^{2}\left(|g|^{2} d \mu\right)
$$

by $U^{\prime} h=h$ for all $h \in P^{2}\left(|f|^{2} d \mu\right)$. It follows from Lemma 1 that $\left(N_{z}, L^{2}\left(|f|^{2} d \mu\right)\right)$ (resp. $\left.\left(N_{z}, L^{2}\left(|g|^{2} d \mu\right)\right)\right)$ is the minimal normal extension of $N_{z} \mid P^{2}\left(|f|^{2} d \mu\right)$ (resp. $N_{z} \mid P^{2}\left(|g|^{2} d \mu\right)$ ). Thus $U^{\prime}$ extends to a unitary operator $V: L^{2}\left(|f|^{2} d \mu\right) \rightarrow L^{2}\left(|g|^{2} d \mu\right)$ which satisfies $V \mid P^{2}\left(|f|^{2} d \mu\right)=U^{\prime}$ and $N_{z} V=V N_{z}$ (refer to Proposition III.2.5 of [3]). It follows that

$$
\int|h|^{2}|f|^{2} d \mu=\int|h|^{2}|g|^{2} d \mu \quad \forall h \in L^{2}\left(|f|^{2} d \mu\right) .
$$

This implies that $|f(\lambda)|=|g(\lambda)|$ a.e. $[\mu]$ on $\Omega^{-}$, the support of $\mu$. If we let $Z(g)=\{\lambda \in \Omega: g(\lambda)=0\}$, then $f / g$ is an analytic map on the open and connected set $\Omega \backslash Z(g)$, which has modulus one a.e. [ $\mu$ ]. Set $\varphi=f / g$ and $\mathscr{U}=\Omega \backslash Z(g)$. Then $\varphi$ is analytic on $\mathscr{U}$ and the closure of $\mathscr{U}$ equals the support of $\mu$.

We claim that $|\varphi(\lambda)|=1$ for all $\lambda \in \mathscr{U}$. To verify this we note that if $\varphi$ is constant, then clearly $|\varphi(\lambda)|=1$ for all $\lambda \in \mathscr{U}$. Thus we may assume that $\varphi$ is not constant. By the open mapping principle $\varphi(\mathscr{U})$ is an open set. This implies that the set

$$
\mathscr{V}=\varphi^{-1}[\varphi(\mathscr{U}) \backslash \mathbf{T}] \quad(\text { where } \mathbf{T}=\{z \in \mathbf{C}:|z|=1\})
$$

is open and contained in $\mathscr{U} \subseteq$ support of $\mu$. But $\mathscr{V}$ has measure 0 since $|\varphi|=1$ a.e. $[\mu]$, hence $\mathscr{V}=\varnothing$. This establishes the claim.

It follows immediately from the above claim and the open mapping principle that $\varphi$ is constant, hence $f=\varphi g \in \mathscr{N}$. 
It is easy to see from Theorem III.8.16 of [3] that the operators $\left(M_{z}, D_{\alpha}\right)$ satisfy the hypothesis of Theorem 1 for $\alpha<0$.

3. The Dirichlet space. Before we state our result about unitarily equivalent invariant subspaces of the Dirichlet shift we shall prove a lemma which shows how the unitary equivalence must be given.

Lemma 2. Suppose $\mathscr{M}, \mathscr{N} \in \operatorname{Lat}\left(M_{z}, D\right)$ are unitarily equivalent.

Then the unitary operator $U: \mathscr{M} \rightarrow \mathcal{N}$ that establishes the unitary equivalence is of the form $U=M_{\varphi}$, where $\varphi$ is the quotient of two inner functions.

Proof. In [5] it was shown that for every $\mathscr{L} \in \operatorname{Lat}\left(M_{z}, D\right)$ and every $\lambda \in \mathbf{D}$ (the unit disc) one has

$$
\operatorname{dim}(\mathscr{L} \ominus(z-\lambda) \mathscr{L})=\operatorname{dim} \operatorname{ker}\left(\left(M_{z}-\lambda\right) \mid \mathscr{L}\right)^{*}=1 .
$$

The arguments given in the proofs of Lemmas 4 and 6 of [6] thus imply that $U=M_{\varphi}$ for some complex-valued function $\varphi$ in the unit disc.

We may assume that $\mathscr{M} \neq\{0\}$. So let $f \in \mathscr{M}, f \neq 0$ and set $g=\varphi f$. $M_{\varphi}$ is unitary, thus

$$
\begin{aligned}
\left(z^{n-1} f, f\right)_{D} & =\left(z^{n-1} g, g\right)_{D} \quad \forall n \in \mathbf{N} \quad \text { and } \\
\left(z^{n} f, z f\right)_{D} & =\left(z^{n} g, z g\right)_{D} \quad \forall n \in \mathbf{N} .
\end{aligned}
$$

On the other hand for $n \in \mathbf{N}$ and for $h(z)=\sum_{k=0}^{\infty} \hat{h}(k) z^{k} \in D$ one has

$$
\begin{aligned}
\left(z^{n} h, z h\right)_{D} & =\left(\sum_{k=n}^{\infty} \hat{h}(k-n) z^{k}, \sum_{k=1}^{\infty} \hat{h}(k-1) z^{k}\right) \\
& =\sum_{k=n}^{\infty}(k+1) \hat{h}(k-n) \overline{\hat{h}(k-1)} \\
& =\sum_{k=n-1}^{\infty}(k+2) \hat{h}(k-(n-1)) \overline{\hat{h}(k)} \\
& =\left(z^{n-1} h, h\right)_{D}+\left(z^{n-1} h, h\right)_{H^{2}} .
\end{aligned}
$$

Comparison of the terms in (1) and (2) with equation (3) yields

$$
\left(z^{n-1} f, f\right)_{H^{2}}=\left(z^{n-1} g, g\right)_{H^{2}} \quad \forall n \in \mathbf{N} .
$$


Note that $D \subseteq H^{2}$. Thus (4) implies that the boundary value functions of $f$ and $g, \hat{f}$ and $\hat{g}$, satisfy

$$
\int_{0}^{2 \pi} e^{\mathrm{int}}\left|\hat{f}\left(e^{i t}\right)\right|^{2} d t=\int_{0}^{2 \pi} e^{\mathrm{int}}\left|\hat{g}\left(e^{i t}\right)\right|^{2} d t \quad \forall n \in \mathbf{Z} .
$$

It follows that $|\hat{f}|=|\hat{g}|$ a.e. on $\mathbf{T}$, hence $f$ and $g$ have the same outer factor. But $g=\varphi f$, thus $\varphi$ must be the quotient of two inner functions.

Theorem 2. Suppose $\mathscr{M}, \mathscr{N} \in \operatorname{Lat}\left(M_{z}, D\right)$ are unitarily equivalent.

(a) If $\mathscr{M}$ contains an outer function, then $\mathscr{M}=\mathscr{N}$.

(b) If $\mathscr{N} \subseteq \mathscr{M}$, then $\mathscr{M}=\mathscr{N}$.

Proof. Suppose the unitary equivalence is given by $M_{\varphi}: \mathscr{M} \rightarrow \mathscr{N}$, where $\varphi$ is the quotient of two inner functions. Note that for all $f \in D$

$$
\|f\|_{D}^{2}=\|f\|_{H^{2}}^{2}+\left\|f^{\prime}\right\|_{2}^{2}
$$

where $\left\|f^{\prime}\right\|_{2}$ is the $L_{a}^{2}$ norm of the derivative of $f$.

We have $\|\varphi f\|_{D}=\|f\|_{D}$ and $\|\varphi f\|_{H^{2}}=\|f\|_{H^{2}}$ for all $f \in \mathscr{M}$. Thus $\left\|(\varphi f)^{\prime}\right\|_{2}=\left\|f^{\prime}\right\|_{2}$ for all $f \in \mathscr{M}$.

(a) If $\mathscr{M}$ contains an outer function $f$, then $\varphi f \in D$ implies that $\varphi$ is an inner function. If $\varphi$ is not a constant, then it follows from Carleson's representation for the Dirichlet integral, [2], that $\left\|(\varphi f)^{\prime}\right\|_{2}>$ $\left\|f^{\prime}\right\|_{2}$. Hence $\varphi$ is a constant and $\mathscr{M}=\mathscr{N}$.

(b) If $\mathscr{N} \subseteq \mathscr{M}$, then $M_{\varphi}$ multiplies $\mathscr{M}$ into itself. By Lemma 11 of [4] $\varphi$ must be bounded on $\mathbf{D} \backslash Z(\mathscr{M})$, where $Z(\mathscr{M})$ is the intersection of the sets $Z(f), f \in \mathscr{M}$. Being the quotient of two inner functions, $\varphi$ must in fact be an inner function. The same reasoning as in part (a) of this proof now yields that $\mathscr{M}=\mathscr{N}$.

The author feels almost certain that the conclusion of Theorem 2 is valid for all unitarily equivalent subspaces $\mathscr{M}, \mathscr{N} \in \operatorname{Lat}\left(M_{z}, D\right)$.

\section{REFERENCES}

[1] O. Agrawal, D. Clark and R. Douglas, Invariant subspaces in the polydisk, Pacific J. Math., 121, No. 1 (1986), 1-11.

[2] L. Carleson, $A$ representation formula for the Dirichlet integral, Math. Z., 73 (1980), 190-196.

[3] J. Conway, Subnormal operators, Res. Notes in Math., 51 (1981).

[4] P. Duren, B. Romberg and A. Shields, Linear functionals on $H^{p}$ spaces with $0<p<1$, J. Reine Angew. Math., 238 (1969), 32-60. 
[5] S. Richter and A. L. Shields, Bounded analytic functions in the Dirichlet space, submitted to Math. Z.

[6] A. L. Shields and L. J. Wallen, The commutants of certain Hilbert space operators, Indiana Univ. Math. J., 20 (1971), 777-788.

Received August 20, 1986. This work appeared as part of the author's Ph.D. dissertation at the University of Michigan (1986). The author would like to acknowledge the many helpful conversations he has had with his advisor Allen Shields.

UNIVERSITY OF VIRGINIA

Charlottesville, VA 22903-3199 


\section{PACIFIC JOURNAL OF MATHEMATICS}

\section{EDITORS}

V. S. VARADARAJAN

(Managing Editor)

University of California

Los Angeles, CA 90024

Herbert Clemens

University of Utah

Salt Lake City, UT 84112

R. FINN

Stanford University

Stanford, CA 94305
HERMANN FLASCHKA

University of Arizona

Tucson, AZ 85721

RAMESh A. GANGOLLI

University of Washington Seattle, WA 98195

VAUGHAN F. R. JONES

University of California

Berkeley, CA 94720
ROBION KIRBY

University of California

Berkeley, CA 94720

C. C. MOORE

University of California

Berkeley, CA 94720

HAROLD STARK

University of California, San Diego

La Jolla, CA 92093

\section{ASSOCIATE EDITORS}
R. AREnS
E. F. BECKENBACH
B. H. NEUMANN
F. WOLF
K. YOSHIDA (1906-1982)

\section{SUPPORTING INSTITUTIONS}

UNIVERSITY OF ARIZONA

UNIVERSITY OF OREGON

UNIVERSITY OF BRITISH COLUMBIA UNIVERSITY OF SOUTHERN CALIFORNIA

CALIFORNIA INSTITUTE OF TECHNOLOGY

UNIVERSITY OF CALIFORNIA

STANFORD UNIVERSITY

MONTANA STATE UNIVERSITY

UNIVERSITY OF HAWAII

UNIVERSITY OF NEVADA, RENO

UNIVERSITY OF TOKYO

NEW MEXICO STATE UNIVERSITY

UNIVERSITY OF UTAH

OREGON STATE UNIVERSITY

WASHINGTON STATE UNIVERSITY

UNIVERSITY OF WASHINGTON 


\section{Pacific Journal of Mathematics}

\section{Vol. 133, No. $1 \quad$ March, 1988}

John Anderson, Finitely generated algebras and algebras of solutions to partial differential equations $\ldots \ldots \ldots \ldots \ldots \ldots \ldots \ldots \ldots \ldots \ldots \ldots \ldots$

Junichi Aramaki, On an extension of the Ikehara Tauberian theorem . . . . . 13

Giacomo Monti Bragadin, Abstract Riemannian stratifications .......... 31

Lawrence James Brenton and Richard Hill, On the Diophantine equation

$1=\sum 1 / n_{i}+1 / \prod n_{i}$ and a class of homologically trivial complex

surface singularities .................................41

C. Bruce Hughes, Controlled homotopy topological structures $\ldots . \ldots \ldots \ldots 69$

Peter Wilcox Jones and Takafumi Murai, Positive analytic capacity but zero Buffon needle probability . ...........................999

Gary M. Lieberman, Hölder continuity of the gradient at a corner for the

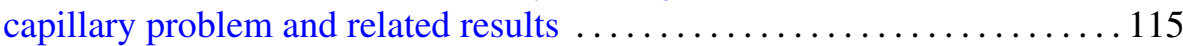

Feng Luo, Representing homology classes of $C \mathbf{P}^{2} \overline{C \mathbf{P}^{2}}$

Claudio Nebbia, Groups of isometries of a tree and the Kunze-Stein

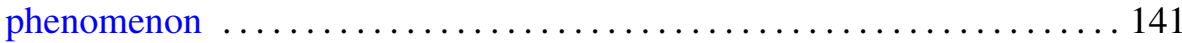

Stefan Richter, Unitary equivalence of invariant subspaces of Bergman and

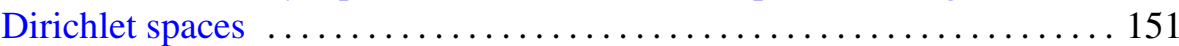

Paul Frederick Ringseth, The Selberg trace formula for groups without Eisenstein series ..................................... 157

Abderrazzak Sersouri, The Mazur property for compact sets $\ldots \ldots \ldots \ldots 185$ Alladi Sitaram, On an analogue of the Wiener Tauberian theorem for symmetric spaces of the noncompact type 